\title{
La Torre del Grau Vell en la defensa de la costa de Sagunto Teresa Gil Piqueras ${ }^{\mathrm{a}}$, Pablo Rodríguez-Navarro ${ }^{\mathrm{b}}$
}

\author{
Instituto de Restauración del Patrimonio, Universitat Politècnica de València, Valencia, Spain
}

a tgil@ega.upv.es; ${ }^{b}$ rodriguez@upv.es

\begin{abstract}
The Grau Vell tower, located in the municipality of Sagunto, is a part of a defensive group developed between the XVI and XVIII centuries for protecting and defending the coast of the Valencian Kingdom. The geographic features of the place and the Confirmation in 1459 of Grau as the unique harbor to ship products of Murviedro, are the key of the election this place as strategic, documental and graphic point to analyze the real state of the tower and the battery. The work methodology is based on photogrammetric draws of the set as the base of constructive analyzing and its materials. The results allow us to determine the current state of the set and they will give us information to contrast with coastal towers.
\end{abstract}

Keywords: torre, fortín, batería a barbeta, Sagunto.

\section{Introducción}

El Fortín o Torre del Grau Vell de Sagunto, también conocida como Torre del Grao de Murviedro o del Grau de Morvedre ${ }^{1}$, forma parte de un singular complejo defensivo compuesto por varias estructuras, construidas entre los siglos XVI y XVIII.

La construcción de la torre como punto estratégico de defensa y control del litoral es fruto de la gran inseguridad marítima que sufrió la costa levantina, especialmente durante los siglos XVI y XVII, que obligó a tomar medidas concretas ante los ataques de corsarios llegados desde el Norte de África. A partir de que el rey Juan II de Aragón, el 14 de marzo de 1459 autorice al Grao Vell como único puerto de embarque de mercancías de la costa de Murviedro, esta zona se hace todavía más vulnerable al ataque de piratas, necesitando de una correcta defensa que le hará al mismo tiempo convertirse en un importante punto de control dentro de la red de torres de vigilancia y defensa del litoral valenciano ${ }^{2}$.
Los primeros testimonios hacen referencia a la necesidad de construir almacenes y oficinas para el fisco, y a la obligación de la defensa y vigilancia de la costa. Esto llevará a que en el siglo XV se construya un cercado de tapia de piedra, sobre el que ya en el siglo XVI se adosará a su muro Este la torre, ejecutada con mampostería y sillares. Durante los siglos XVII y XVIII se completará la estructura con la construcción de una batería a barbeta situada al Este de la torre, rodeada por un foso, y la construcción de varios almacenes y casas. Hoy en día es muy difícil acceder a esta torre, que ha quedado prácticamente aislada dentro de sus lindes que son al Norte, el parque industrial de Sagunto, y al Sur y al Oeste, la Marjal dels Moros considerada Zona de Especial Protección de Aves (ZEPA).

El Fortín de Grau Vell fue declarado Bien Inmueble de Interés Cultural, en la categoría de monumento en 2010, por la Generalitat Valenciana (R-I-51-0010971). 


\section{Situación y acceso a la torre}

El Barrio del Grau Vell se encuentra en la Partida de la Vila, a seis kilómetros de distancia del centro de Sagunto, al Sur del parque industrial. A pesar de su proximidad a Sagunto, el acceso es complicado, teniendo que cruzar todo el polígono industrial, situado junto al puerto comercial de Sagunto para acceder a él. A medida que nos acercamos al Grau por una estrecha carretera asfaltada, lo primero que vemos es la torre y su batería $\left(39^{\circ} 38^{\prime} 06^{\prime \prime} \mathrm{N}\right.$, $\left.0^{\circ} 14^{\prime} 23^{\prime \prime} \mathrm{O}\right)$.

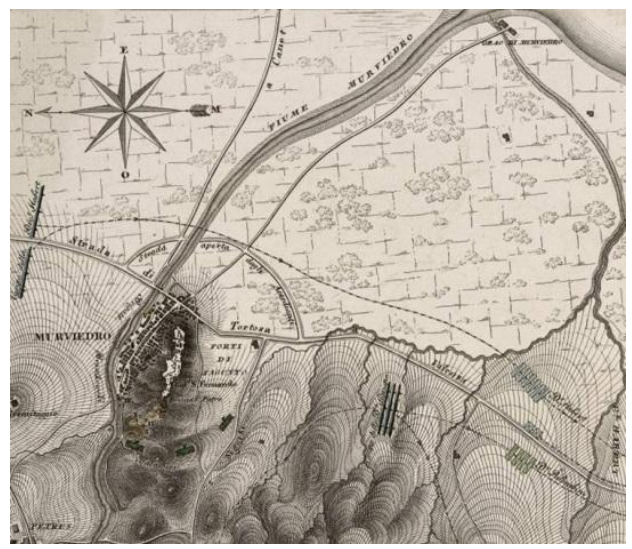

Fig. 1- Mapa de Murviedro con la situación del Grau Vell al Norte. (SG. Ar.G-T.3-C.2-246, 1811)

Este conjunto defensivo se encuentra situado en primera línea, a poco menos de $40 \mathrm{~m}$. de la línea actual de costa, en una zona llana de marjal, a apenas $1 \mathrm{~m}$. por encima del nivel del mar.

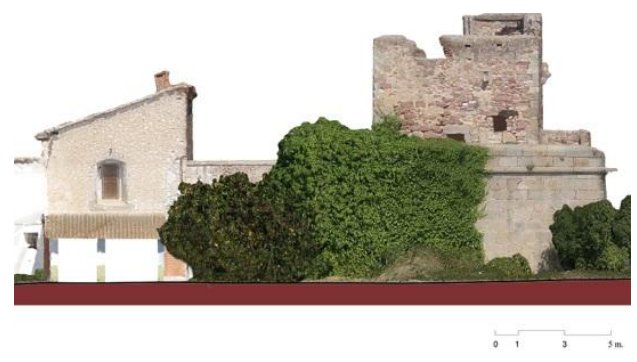

Fig. 2- Alzado Fotogramétrico de la fachada Este del Fortín (Rodríguez-Navarro \& Gil Piqueras, 2015)
En su parte Norte y Sur la torre está rodeada de casas de poca altura, antiguos almacenes y estancias, conservando en su parte trasera el antiguo cercado construido anteriormente a la torre, y su pozo. Los almacenes situados al Norte, están completamente abandonados y sin uso, lo que les hace estar en un estado de ruina bastante avanzado, mientras que la nave situada al Sur se encuentra ocupada y en buen estado, gracias al uso responsable de sus propietarios.

\section{Antecedentes históricos}

Los acuerdos llevados a cabo en las Cortes de Monzón de 1528, 1534 y 1547 coinciden en la necesidad de proteger la costa de las sucesivas incursiones de turcos y berberiscos. En ellas se insiste en el modo de hacer frente a estas incursiones, cada vez más seguidas y violentas, y se establecen criterios para proteger el litoral y crear una guardia costera. Incluso se cambia la estrategia en la defensa, siendo por primera vez en las Cortes de Monzón de 1547, cuando la idea de fortificar el litoral reemplazó a la corriente tradicional que perseguía la protección, sin mucho éxito, de la costa con galeras dotadas de una armada regular bajo la dirección del Emperador Carlos V. (Arcinega, 1999)

A pesar de ello los ataques continúan y es en las Cortes de Monzón de 1552 cuando se toman medidas más contundentes. Las tres instituciones principales del Reino de Valencia (real, militar, y eclesiástica) se unen y aprueban la construcción de torres y atalayas en el litoral levantino. Las obras comienzan en Valencia y se financian gracias a un impuesto que se fija sobre la seda que se saca del reino (Salvador, M.D. 1997). Según la misma autora en 1553/54, los jurados de Murviedro reciben órdenes del Virrey de Valencia D. Bernardino de Cárdenas y Pacheco, Duque de Maqueda, para que cooperen en la defensa del reino, reforzando la muralla de la villa y de sus torres costeras. Esta época marca el inicio de la construcción/adecuación de las torres vigía como elementos dentro de un sistema global y organizado de defensa de la costa, proyecto que retomaría Felipe II tras ascender al trono en 1556, implantándolo en todo el territorio. 


\subsection{Primeras referencias a la torre del Grau Vell de Murviedro}

En 1534 la villa de Murviedro aceptó el plan del virrey Fernando de Aragón de fortificar $y$ reparar las murallas de la ciudad, y de sus torres y fortines de la costa, tal y como quedó reflejado en el "Manual de Consells de la Vila de Morvedre", hoy desaparecido tras un incendio en el Archivo Municipal de Sagunto (Chabret, 2002). El compromiso que adquirió la villa ante Juan Cervelló, comisario especial para este efecto en todo el reino, fue el de ejecutar las obras en 7 meses. A pesar de ello no hay ninguna referencia a la existencia de la Torre del Grau Vell hasta avanzada la segunda mitad del siglo XVI, no apareciendo hasta esa época ni en mapas históricos, ni siquiera en el informe del Duque de Maqueda de 1554.

En 1563, en el "Discurso sobre la fortificación y defensa del Reino de Valencia,...," que el ingeniero militar Giovanni Battista Antonelli hace a petición de Felipe II, escribe: Morviedro se fortificara assi mismo y el castillo se pondrá en buena defensa. Tras este proyecto, en 1585 Juan de Acuña describe por primera vez a la torre, diciendo de ella en su informe: La torre del Grao de Morvedre,..., es quadrada y hasta la media está terraplenada, y tiene unas troneras buenas, y una garita que guarda la puerta, a la qual se sube por una escala de mano, ay en ella una pieça de artillería que tira pelota como naranja, tiene para ella una dozena de pelotas y arrova y media de pólvora, y arrimada a la dicha torre ay un reducto grande en donde ay magazenes para lo que se trae a cargar al dicho grao y se descarga en él, ....

En este informe Acuña cita la existencia de un recinto cerrado dotado de almacenes en donde guardar las mercancías en espera de ser embarcadas, aunque hoy en día no se conservan. Respecto a la entrada a la torre, en esta época está situada en el muro Norte, sobre elevada y bajo un recercado de piedra del que todavía quedan restos. Vemos que en el informe también se hace referencia al sistema defensivo y a su guardia cuando dice, ,...y en la torre ay dos guardas con arcabuzes, y en el reducto dos atajadores de a cavallo que atajan hasta la torre del Puche y desde esta torre a la del rio de Morvedre,... De la interpretación del texto se deduce que la guardia costera de la Torre del Grau Vell estaba formada por un total de cuatro hombres, dos guardas y dos atalladors.

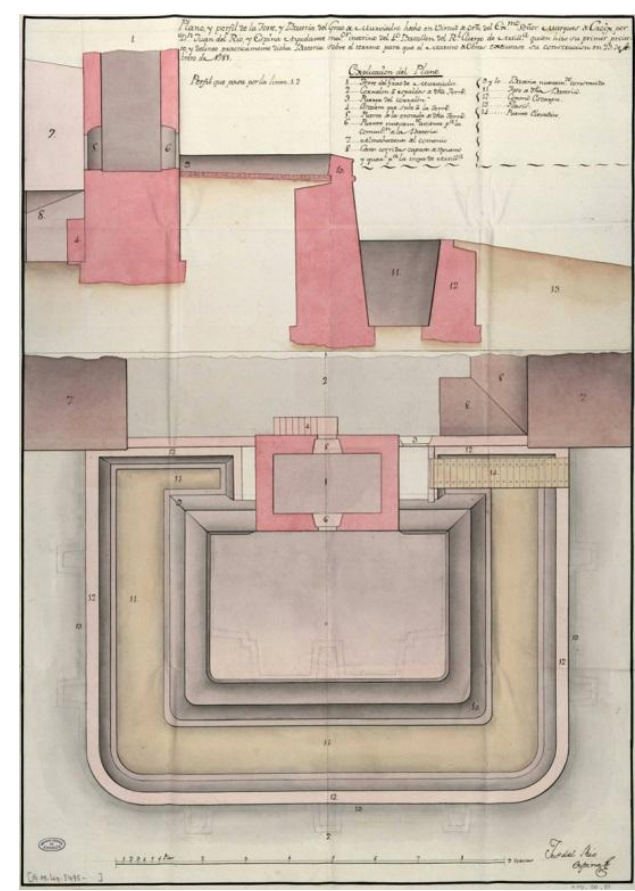

Fig. 3- Sección del Fortín (Del Río y Espina, 1781. A.G.S. MPD, 50, 089)

Este hecho cambia en las "Ordinacions tocants a la custodia y guarda de la costa marítima del Regne de Valencia", redactadas en 1673 por Vespasiano Manrique, Conde de Paredes. En ellas establece el mismo personal, pero los atalladors ya no van los dos a la Torre del Puig, sino que uno irá hasta un punto llamado Fornas donde se encontrará con el atallador de la Torre de Canet, y el otro irá hacia el Sur hasta la acequia de Puzol, donde se encontrará con el atallador de la Torre del Puig.

La construcción de la batería adosada a la torre es muy posterior y es consecuencia de la Real Orden de S.M. Carlos III de 1780, por la que se encarga el proyecto de ejecución de la batería que completa el fortín, tal y como aparece en los planos que envían D. Juan Verchera y Platet, subdelegado de marina, y el Marqués de Croix a 
D. Miguel Múzquiz, Señor de Murviedro, en 1780 y 1781 respectivamente (AGS. Secretaría de Guerra, MPD, 50,082 y 50,088). En estos planos se ve la torre situada frente a un cercado, que aparece rodeada por tres de sus cuatro lados por una batería dotada de un foso.

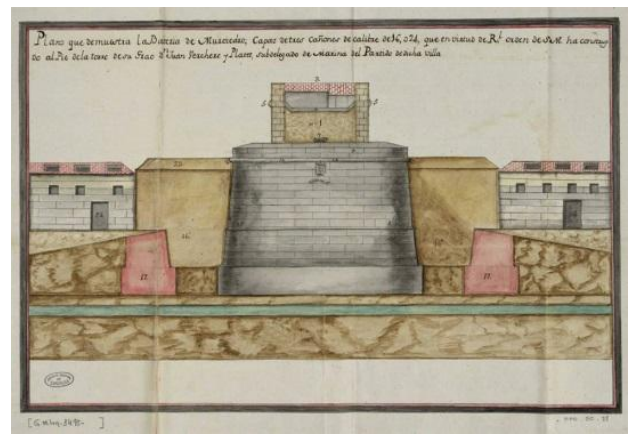

Fig. 4- Plano de 1781 en donde se muestra el alzado de la batería de Murviedro, torre, foso y almacenes (A.G.S. MPD, 50, 088)

En el año 1788, D. José de Roxas, comandante general del Reino de Valencia, en su "Relación circunstanciada de la consistencia de la costa marítima de los Reinos de Valencia y Murcia" incluye una descripción muy completa del conjunto defensivo, diciendo: está situada esta torre en llanura sobre la playa de su nombre a tiro de fusil del Mar, sirve de resguardo a la costa, y a varios almacenes de particulares, en que depositan sus frutos para embarcarlos en los buques de comercio que acuden a dicha playa con tiempos bonancibles, su figura es un quadrilongo, cuyo lado menor tiene 5 varas, y 2 pies, y el mayor ocho varas, con 12 y $1 / 2$ de alto. Esta terraplenado hasta su mitad, y se sube por una escalera de cantería que tiene adosada por la parte de tierra hasta la altura de 3 varas y 1/2 en que forma un rellano, y desde él a la puerta de la torre hay una escalera de mano de 3 varas de alto: dicho primer piso se sube por escalera de madera a la plaza de armas que tiene su tinglao y ladronera para defender la puerta; a las espaldas de esta torre hay un grande corral, o cercado que en medio tiene un pozo, y a la parte de Levante 4 habitaciones, una de ellas de repuesto de pólvora, y las otras para alojamiento de su guarnición.
Como podemos ver en esta época el acceso a la torre ha cambiado, estando situado en el muro Oeste, recayendo dentro del cercado. Del mismo modo, con esta descripción vemos que ya estaban construidos los almacenes laterales a la torre, y la batería adosada a su muro Este. Respecto a la defensa, en el mismo documento Roxas aconseja subir el número de personas que custodian la torre al decir: Guarnecen este puesto dos torreros, y dos atajadores que alternan por mitad en el servicio, dicha guarnición devería aumentar en tiempo de paz con un artillero para el cuidado de los cañones, y demás pertrechos en de guerra reforzarse con un cabo, y tres artilleros mas, 12 paysanos para ayudar al servicio de la artillería.

\section{Descripción formal y constructiva}

La construcción del fortín del Grau Vell ha sido el resultado de la evolución del conjunto arquitectónico a lo largo del tiempo, como consecuencia de la adaptación a las necesidades defensivas del momento, de manera que hoy en día está formado por un cercado, almacenes, una torre y una batería dotada de un foso. Las distintas etapas en las que se construyó el fortín, van desde el siglo XVI al siglo XVIII (Aleixandre, 1989). A continuación pasamos a describir cada uno de los elementos que componen este fortín.

\subsection{Cercado posterior y almacenes}

Como hemos podido comprobar el cercado fue la primera parte en ejecutarse. Estaba formado por un espacio de planta sensiblemente cuadrada, cerrado por un muro, en cuyo interior había unas estancias y un pozo, aún visible. Aunque la construcción del cercado está fijada en el siglo XVI, hay quien opina que es anterior, ya que al ser el único embarcadero de la zona debía estar dotado de un espacio cerrado en donde guardar las mercancías antes de ser embarcadas. José de Roxas lo describe de la siguiente manera: a las espaldas de esta torre hay un grande corral, o cercado que en medio tiene un pozo, y a la parte de Levante 4 habitaciones, una de ellas de repuesto de pólvora, y las otras para alojamiento de su 
guarnición. Este cercado se puede ver, aunque proporcionalmente con una dimensión menor, en el plano de 1780 del A.G.S., MPD, 50, 082. En la actualidad este espacio está ocupado en gran parte por corrales y pequeños trasteros de los vecinos de las casas adyacentes. La entrada al interior del cercado era a través de una puerta con dintel arqueado practicada en su muro Este, de la que solo queda una jamba y parte de uno de los arranques del arco.

Constructivamente si nos fijamos, aún podemos ver en la fábrica actual restos del muro original que componía este cercado, así como diferentes fases de ejecución. Así en el muro situado en prolongación de la torre observamos dos fases de ejecución en la fábrica, que nos marcan dos alturas, existiendo una primera de $3,70 \mathrm{~m}$. de altura correspondiente posiblemente al primer cercado, que terminó sobre elevándose posteriormente hasta llegar a los $5,30 \mathrm{~m}$. de altura, tal y como vemos hoy en día. En general, este muro está ejecutado en su primera fase con tapial de mortero de cal y cantos bien ordenados, sobre cimentación de grandes mampuestos, estando en su segunda fase, de menor sección que la anterior, ejecutado con tapial de tierra y gravas.

Con el paso de los siglos, los muros del cercado han sufrido diversas actuaciones. En la fábrica del muro Este observamos que para la construcción de la torre se degolló parte del cercado sustituyéndose por una nueva fábrica, más resistente y de mejor calidad, sobre la que se construyó la torre. También se ve que los muros de sus fachadas Norte y Sur se aprovecharon para apoyar sobre ellos los cerramientos exteriores de los almacenes. El acceso también se ha modificado, ya que durante los años 70 del pasado siglo se abrió un nuevo hueco de acceso, situado también en su muro Este, pero en este caso al Sur de la torre.

La construcción de los almacenes que actualmente vemos adosados a la parte exterior de los muros del cercado, se hizo en dos fases datadas en el siglo XVII y XVIII respectivamente. En este periodo sobre las fachadas Norte y Sur del cercado se adosaron naves destinadas a almacén. Del mismo modo y tal y como vemos en el plano del A.G.S., MPD, 50, 082, de 1780, en su interior habían unas estancias, de una altura, que cumplían la función de cuartel de artilleros, almacén para la pólvora y cocinas. No podemos afirmar con certeza si estas mismas estancias que vemos en este plano, estaban construidas antes de los almacenes o son coetáneas a ellos, a pesar de que Acuña en su informe nos dice: desde la construcción del cercado existieron en su interior estancias en donde guardar las mercancías... Al respecto nosotros más bien nos inclinamos a pensar que estas estancias fueron una evolución de las originales, de uso privado, y en las que los mercaderes guardaban sus productos en espera a ser embarcados o bien resguardaban las mercancías llegadas del exterior, en espera de ser distribuidas por el reino.

Respecto a los almacenes situados fuera del cercado, sabemos que se construyeron en dos periodos: los situados al Sur son de 1607 aproximadamente, y los del Norte datan de 1711 , tal y como aparece tallado en un dintel situado sobre uno de los accesos.

Los almacenes situados al Norte, están construidos con sillería, en dos tercios de su altura, y mampostería ordinaria el último tercio, presentando varios huecos a la altura de planta baja, actualmente cegados. En general son de una altura, a excepción del primer módulo más próximo a la torre, que tiene dos alturas. $\mathrm{Su}$ cubierta es a dos aguas con vigas y viguetas de madera a par y picadero. En la parte Sur solo queda el primer módulo de almacén, conservando dos alturas, rematadas con una cubierta a un agua.

\subsection{Torre del Grau Vell}

La torre fue construida entre 1562 y 1563 (Aleixandre, 1989), ejecutándose en medio del muro del cercado recayente al mar. Fue el segundo elemento del completo defensivo en construirse, dando protección al recinto cerrado frente a los ataques llegados desde la costa. $\mathrm{Al}$ observar hoy día le torre vemos que se trata de una estructura de planta cuadrangular, de $6,00 \mathrm{x}$ 7,00 m. de lado aproximadamente, ejecutada con muros de mampostería y sillares en las esquinas, 
puertas y ventanas. Tiene una altura total de 10,70 m., que se elevan un metro más en la zona Noroeste, donde tiene una garita.

La torre consta de dos alturas. La planta baja se encuentra rellena en su interior con cantos de rio y mortero de cal, mezclado con arenas y gravas de diversos tamaños, lo que se puede ver en algunas zonas gracias a la pérdida de mampuestos de su cerramiento. Este recurso aportaría resistencia a la estructura, a la vez que contribuiría en su momento a facilitar su proceso constructivo. Además en este caso las necesidades de uso de la planta baja de la torre, tales como cuadra para los caballos de los atalladors, almacén o aljibe, que normalmente se busca en otras torres, quedan cubiertas con las estancias del cercado posterior, no siendo necesario su uso. En la primera planta, en la que se alojarían los atalladors, es en donde se sitúa la única sala, cerrada por una bóveda de cañón hecha a base de ladrillos. Desde esta planta se accede directamente a la plataforma de la batería y a la terraza, a la que se llega a través de un hueco practicado en la esquina Sureste de la bóveda, accesible únicamente mediante una escalera de mano.

En la torre pueden distinguirse claramente dos tipos de mampostería. La fábrica de la parte inferior está ejecutada con mampuestos grandes y bien ordenados recibidos con mortero de cal, mientras que la parte superior está ejecutada con mampostería ordinaria, de muy distintos tamaños, recibidos con mortero de cal mezclado con arenas y gravas. En los parapetos de cerramiento de la torre, vemos que éstos varían su espesor de 0,50 m. a 0,85 m., siendo más gruesos los recayentes al Norte y al Este. Es posible que esto sólo se dé en este nivel, debiendo ser los muros en su parte inferior de igual espesor. La ejecución de los muros se debió realizar hilada por hilada, subiendo todos los muros al mismo tiempo, garantizando la traba entre unas hiladas y otras, tanto longitudinalmente como transversalmente. Para esquinas y huecos se utilizaron sillares, y el matacán se ejecutó con sillarejos.

En cuanto a la terraza de la torre, es plana sobre una bóveda de cañón de ladrillos colocados a rosca, tomados con mortero de cal. La bóveda debió ejecutarse sin ninguna cimbra, apoyando en los muros las hiladas de ladrillo que van inclinadas respecto a la horizontal un ángulo aproximado de 40 grados, de forma que el rozamiento con la hilada anterior la va manteniendo estable en su ejecución sin necesidad de cimbra. Por su parte superior, el pavimento de la terraza presenta una ligera inclinación hacia las fachadas Este y Oeste, por donde desagua a través de gárgolas de piedra. Una vez en la terraza, un habitáculo de $2,30 \mathrm{x}$ $3,30 \mathrm{~m}$. de lado se eleva en su esquina Noroeste, generando una garita, denominada garitón en los planos del proyecto de ejecución de la batería de 1780 y 1781 . Este garitón estaba cubierto por un tejadillo ejecutado sobre estructura de madera, del que aún quedan restos de rollizos.

En el muro Norte de la torre observamos restos de la entrada original, del que sólo queda el hueco enmarcado por sillares y restos del matacán desde el que defenderlo. Esta entrada quedó sin uso cuando se ejecutó la batería. En el mismo muro hay dos troneras con un pronunciado derrame y poco abocinamiento, situadas al nivel de la estancia y a la altura de la terraza. En el muro Oeste, a la altura también de la primera planta encontramos la segunda entrada consecuencia de la ejecución de la batería. A esta entrada se llegaba con una escalera de mano que se apoyaba sobre un rellano situado a mitad de su altura, al que se accedía a través de una escalera de piedra construida paralela a la torre, y de un único tramo. En este caso la entrada tiene un dintel compuesto de dos sillarejos de rodeno, siendo sus jambas de mampostería, a excepción de una pequeña parte coincidente con una tronera compuesta por sillares que se adhiere a la entrada por su lado Sur. En la misma fachada, a la altura del suelo de la estancia, se disponen otras dos troneras más pequeñas y con mucho más derrame que la anterior, facilitando el disparo a nivel del suelo. Sus jambas y dinteles debían ser de mampuestos a pesar de que hoy en día una de ellas, fruto de alguna reparación, presenta un dintel arqueado a base de ladrillo.

Respecto a los otros dos muros, vemos que el muro Sur tiene una tronera abocinada y con poco 
derrame, quedando por su izquierda adosado el muro del cercado posterior. Finalmente en el muro orientado al Este, recayente al mar, y a la altura de la primera planta, se encuentra el hueco de salida a la terraza rematado por un dintel de piedra, y una ventana con dintel de sillarejo de rodeno y jambas de mampostería con algún sillar. Todos estos huecos se ejecutaron cuando se construyó la batería. En su parte superior, en el peto que cierra la terraza, una tronera se abre hacia el Este con abocinamiento hacia el exterior, dando posible servicio a una pieza de artillería. Dados los distintos materiales que hoy en día aparecen rodeando este elemento, es posible que en origen la tronera fuera mayor, tal y como se aprecia en los planos del proyecto de ejecución de la batería de 1780 y 1781 .

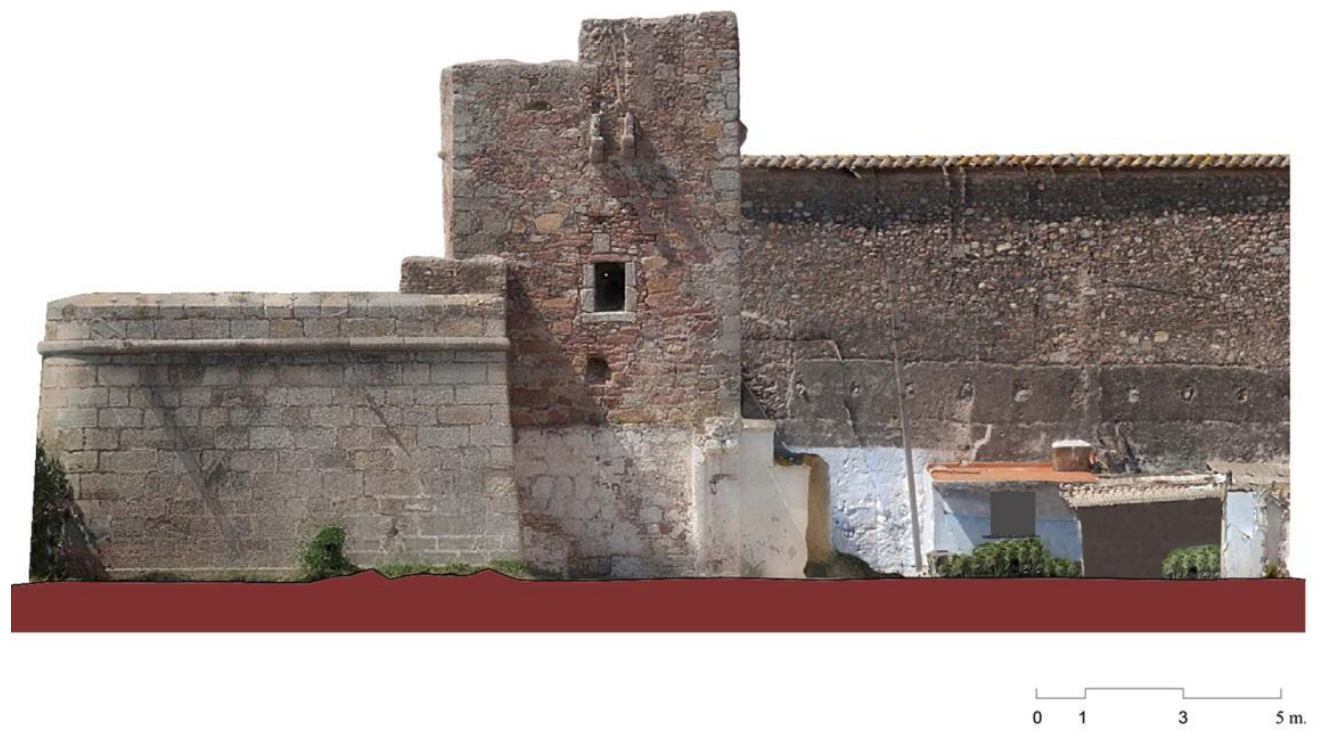

Fig. 6- Alzado fotogramétrico de la fachada Este del Fortín (Rodríguez-Navarro \& Gil Piqueras, 2015)

\subsection{Batería y foso}

La batería está construida frente a la torre, adosada a su muro Este. Su función era la de alojar las piezas de artillería con las que defender el fortín. Su estructura, más baja y ancha que la torre, tiene unos $6,00 \mathrm{~m}$. de altura y $10,50 \times 13,70 \mathrm{~m}$. de base, y está rematada por una azotea plana que desagua al Norte y al Sur a través de dos gárgolas, encontrándose en su interior completamente terraplenada.

Esta batería al igual que la torre, está terraplenada en su interior, y fue diseñada para albergar 3 cañones situados sobre tres estribos, proyectados en las esquinas y centro de la batería. En ella se podían distinguir tres partes: el foso, el muro y la plataforma. El foso estaba precedido por el glacis o explanada que protegía el acceso a la batería. Éste quedaba delimitado por la contraescarpa que es como se denomina al muro de la parte exterior del foso y la escarpa, muro opuesto. Según el proyecto de 1871, como protección a la base del muro encontramos la berma. El muro de la batería se eleva hasta terminar en un parapeto de poca altura, que cierra la plataforma. Este hecho hace que se denomine la batería a barbeta, ya que la poca elevación del muro permite disparar desde la plataforma sin la existencia de merlones o troneras.

Por el exterior, y a la altura del suelo de la plataforma de la batería, un cintón de piedra recorre todo su perímetro, el cual se ve interrumpido en el centro por la presencia de un escudo tallado en piedra. En el muro Norte, sobre el parapeto de la batería y adosado a la torre, se eleva un murete de mampostería muy deteriorado en el que se aprecian restos de dos 
pequeñas aspilleras, que debían usarse para defender la entrada al cercado y el acceso al puente voladizo, que estaba situado en este extremo.

Toda la batería está construida a base de sillares de piedra bien tallados, dando como resultado una fábrica ordenada. Actualmente el foso se ha rellenado y el puente levadizo no existe.

\section{Conclusiones}

La importancia que adquirió Murviedro como enclave comercial hizo que hubiera necesidad de proteger esta parte de la costa. Por este motivo en el siglo XVI se construyó un cercado y una torre desde la que defender y vigilar las posibles incursiones llegadas desde el mar. Con el paso de los años, lo que era una defensa puntual de un enclave comercial se convirtió en un punto estratégico para la defensa de la costa del Reino de Valencia. Años después, la torre pasó a dotarse con una batería a barbeta, en donde alojar las piezas de artillería, precedida por un foso, adaptándose a las necesidades del momento. Como resultado de su evolución, ha llegado a nosotros un completo fortín, al que se adosaron almacenes y estancias.

En general hoy en día la torre y la batería están en buen estado, a pesar de que la torre presenta algunos huecos en sus muros por pérdidas de mampuestos, que necesitarían ser reparados.
Además, la vegetación está afectando cada vez más a sus muros, por lo que debería ser eliminada por completo. Los almacenes no siguen igual suerte, estando en general muy deteriorados. A pesar de ello, vemos que en general la falta de atención y mantenimiento del fortín es completa, requiriendo de un urgente y profundo estudio para su correcta conservación, que garantice su durabilidad y estabilidad a lo largo del tiempo.

\section{Notas}

(1) El topónimo Murviedro es con el que se conocía a la villa existente a los pies del castillo de Sagunto hasta el siglo XIX. A partir de esta época comenzó a constar como Sagunto, pasándose a usar el nombre Murviedro o Morvedre (término en valenciano) para designar a toda la comarca, compuesta por 16 municipios, a cuya cabeza se sitúa la localidad de Sagunto.

(2) Esta aportación se engloba dentro del Proyecto I+D del Programa Estatal de Investigación Científica y Técnica de Excelencia, Subprograma Estatal de Generación de Conocimiento, financiado por el Ministerio de Economía y Competitividad, que lleva por título "Torres de vigía y defensa del litoral valenciano. Generación de metadatos y modelos 3D para su interpretación y efectiva puesta en valor", referencia HAR2013-41859-P.

\section{Referencias}

A.G.S. MPD, 50, 086. MPD, 50, 088. MPD, 50, 082. MPD, 50, 089.

Aleixandre Navarro, J. (1989). "Ubicación de las defensas marítimas en la comarca del camp de Morvedre". ARSE. 24. Sagunto. pp. 871-899.

Antonelli, G.B. (1563). Discurso sobre la fortificación y defensa del Reino de Valencia. en AGS. E, 329-I. doc.13.

Arcinega García, L. (1999). "Defensa a la antigua y a la moderna en el reino de Valencia durante el siglo XVI”. Espacio, Tiempo y Forma. Serie Vil. H. del Arte. t. 12. pp. 61-94.

Boira Maiques, J.V. (2007). Las torres del litoral valenciano. Conselleria de Infraestructuras y Transporte, Valencia.

Chabret Fraga, A. (2002) Sagunto su historia y sus monumentos. Bancaja, D.L. Sagunto. pp. 383-384.

Salvador Lizondo M.D. (1997). "Sagunto, lugar estratégico en la defensa de la costa contra piratas y corsarios». ARSE. Boletín del Centro Arqueológico de Sagunto. 15. pp. 186-187.

SG. Carta dei contorni di Sagunto relativa all'asedio dei Forti, e dalla Bataglia data il 25 Ottobre 1811. Ar.G-T.3-C.2-246. 